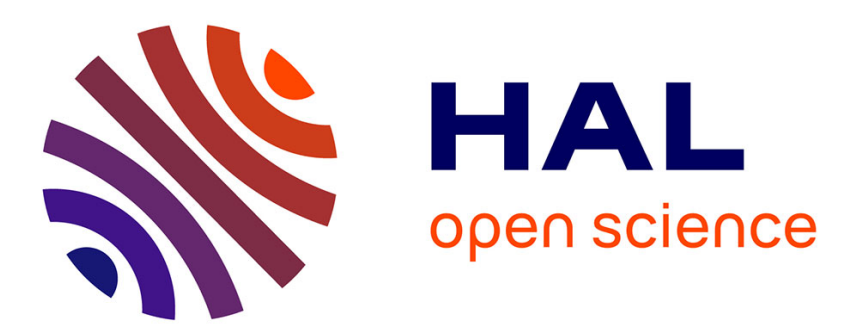

\title{
Les entreprises face à la mutation énergétique: l'enjeu de la mobilité dans les territoires périurbains de la métropole lyonnaise
}

\author{
Patricia Lejoux
}

\section{To cite this version:}

Patricia Lejoux. Les entreprises face à la mutation énergétique: l'enjeu de la mobilité dans les territoires périurbains de la métropole lyonnaise. Revue Géographique de l'Est, 2014. halshs-02491469

\section{HAL Id: halshs-02491469 \\ https://shs.hal.science/halshs-02491469}

Submitted on 26 Feb 2020

HAL is a multi-disciplinary open access archive for the deposit and dissemination of scientific research documents, whether they are published or not. The documents may come from teaching and research institutions in France or abroad, or from public or private research centers.
L'archive ouverte pluridisciplinaire HAL, est destinée au dépôt et à la diffusion de documents scientifiques de niveau recherche, publiés ou non, émanant des établissements d'enseignement et de recherche français ou étrangers, des laboratoires publics ou privés. 
Revue

Géographique de l'Est

\title{
Revue Géographique de l'Est
}

vol. 54 / 1-2 (2014)

Mutations des systèmes productifs en France

Patricia Lejoux

Les entreprises face à la mutation énergétique : l'enjeu de la mobilité dans les territoires périurbains de la métropole lyonnaise

\begin{abstract}
Avertissement
Le contenu de ce site relève de la législation française sur la propriété intellectuelle et est la propriété exclusive de l'éditeur.

Les œuvres figurant sur ce site peuvent être consultées et reproduites sur un support papier ou numérique sous réserve qu'elles soient strictement réservées à un usage soit personnel, soit scientifique ou pédagogique excluant toute exploitation commerciale. La reproduction devra obligatoirement mentionner l'éditeur, le nom de la revue, l'auteur et la référence du document.

Toute autre reproduction est interdite sauf accord préalable de l'éditeur, en dehors des cas prévus par la législation en vigueur en France.
\end{abstract}

revues.org

Revues.org est un portail de revues en sciences humaines et sociales développé par le Cléo, Centre pour l'édition électronique ouverte (CNRS, EHESS, UP, UAPV).

Référence électronique

Patricia Lejoux, «Les entreprises face à la mutation énergétique : I'enjeu de la mobilité dans les territoires périurbains de la métropole lyonnaise », Revue Géographique de l'Est [En ligne], vol. 54 / 1-2 | 2014, mis en ligne le 15 octobre 2014, consulté le 31 octobre 2014. URL : http://rge.revues.org/5135

Éditeur : Association des Géographes de l'Est

http://rge.revues.org

http://www.revues.org

Document accessible en ligne sur :

http://rge.revues.org/5135

Document généré automatiquement le 31 octobre 2014. La pagination ne correspond pas à la pagination de l'édition papier.

Tous droits réservés 


\section{Les entreprises face à la mutation énergétique : l'enjeu de la mobilité dans les territoires périurbains de la métropole lyonnaise}

La raréfaction prévisible des ressources énergétiques fossiles et la nécessité de réduire les émissions de gaz à effet de serre face au défi du changement climatique rendent une mutation énergétique indispensable (Rifkin, 2012). Dans le domaine de la mobilité, cette mutation énergétique constitue un enjeu majeur en raison, d'une part, de la forte dépendance des transports au pétrole et, d'autre part, de leur contribution au réchauffement climatique. Le développement spectaculaire de la mobilité des biens et des personnes à partir de la seconde moitié du $\mathrm{XX}^{\mathrm{e}}$ siècle a été permis par une consommation intensive de pétrole dans un contexte où celui-ci était abondant et bon marché. Aujourd'hui, le secteur des transports est le premier secteur consommateur de pétrole dans le monde représentant à lui seul $62 \%$ de la consommation mondiale de pétrole (IEA, 2013) et il en est extrêmement dépendant puisque $95 \%$ de l'énergie consommée par le secteur des transports provient du pétrole (IEA, 2009). Or, les réserves pétrolières sont amenées à diminuer dans les années à venir, ce qui risque de se traduire par une hausse des prix du pétrole et donc une augmentation du coût de la mobilité. Cette raréfaction est liée au fait que, comme toute énergie non renouvelable, les réserves en pétrole sont limitées : bien que controversé, le peak oil, c'est-à-dire le moment où la production de pétrole conventionnel sera maximale, devrait être atteint autour des années 2020-2030. Mais quels que soient l'importance de ces réserves en pétrole et le moment à partir duquel elles commenceront à décroître, elles se révèleront de toute façon insuffisantes pour faire face à la demande croissante de mobilité portée désormais par les pays en développement, au premier rang desquels figurent la Chine et l'Inde (Orfeuil, 2008). Parallèlement, la mobilité des biens et des personnes contribue de manière significative au réchauffement climatique à travers l'émission de gaz à effet de serre, en particulier de $\mathrm{CO}_{2}$. Le secteur des transports est le deuxième secteur le plus émetteur au monde représentant $22 \%$ des émissions de $\mathrm{CO}_{2}$ mondiales (IEA, 2013) et la réduction de ses émissions constitue, à ce titre, une priorité des politiques publiques de lutte contre le changement climatique, qu'elles soient menées au niveau international, national ou local. La mutation énergétique qui s'annonce dans le domaine de la mobilité nécessite donc de réfléchir au développement d'une offre en transports alternatifs et à une évolution des comportements de mobilité.

Cette mutation énergétique dans le domaine de la mobilité risque de ne pas affecter les entreprises de la même façon, elle apparaît plus problématique pour les entreprises fortement dépendantes de la mobilité routière. C'est le cas notamment des entreprises des secteurs de l'industrie, du commerce de gros et de la logistique pour qui l'accessibilité routière, mais aussi la disponibilité et le prix du foncier sont devenus des critères déterminants de localisation. Ces entreprises ont connu au cours des dernières décennies une évolution de leurs critères de localisation qui s'est traduit par un rejet des localisations centrales (inadaptation des locaux, rareté du foncier, congestion, opposition des riverains...) et un attrait pour les localisations périurbaines (Mérenne-Schoumaker, 2008a et 2008b). L'accessibilité routière a joué un rôle essentiel dans le choix de localisation de ces entreprises dont l'activité est très dépendante de la mobilité : l'implantation dans des territoires périurbains desservis par des infrastructures routières performantes (autoroutes, rocades, voies rapides) leur permet d'être connectées aux flux régionaux, nationaux et internationaux sans pour autant souffrir des problèmes de congestion.

Comment les entreprises implantées dans ces territoires périurbains et dépendantes de la mobilité routière envisagent-elles cette mutation énergétique dans le domaine de la mobilité 
(transport de marchandises, déplacements professionnels, déplacements domicile-travail) ? Est-ce aujourd'hui un sujet de préoccupation pour ces entreprises ou est-ce un enjeu trop éloigné par rapport à leur horizon temporel qui se limite souvent à quelques mois, surtout dans un contexte de crise économique ? Quelles sont les stratégies développées par les entreprises pour faire face à cette mutation énergétique dans le domaine de la mobilité ? Envisagent-elles, par exemple, de diversifier les modes de transport auxquels elles ont recours (transport multimodal, véhicules électriques, biocarburants) ou sont-elles résignées à absorber les hausses du prix du pétrole et donc du coût de la mobilité, celle-ci étant devenue indispensable à leur fonctionnement ? Cette augmentation du coût de la mobilité ne risquet-elle pas de peser sur le budget des salariés de ces entreprises qui, dans ces territoires périurbains, n'ont pas d'autre mode alternatif que la voiture pour se rendre sur leur lieu de travail, surtout dans un contexte où ceux-ci sont de plus en plus éloignés des lieux de résidence (Quételard, 2010) ? Cela peut-il poser à plus long terme des problèmes de recrutement ou de fidélisation de la main d'œuvre pour ces entreprises ? Enfin, quelles pourraient être les conséquences de ces stratégies pour le devenir des territoires périurbains ? Pour répondre à ces questions, nous présenterons les résultats d'une recherche menée auprès d'entreprises implantées dans les territoires périurbains de la région lyonnaise : après avoir exposé la méthodologie de la recherche (I), nous mettrons en évidence les enjeux que cette mutation énergétique dans le domaine de la mobilité représente du point de vue des entreprises (II), puis nous nous intéresserons aux conséquences que ces enjeux peuvent avoir pour les territoires périurbains (III).

\section{Méthodologie}

Les résultats présentés dans cet article sont issus d'une recherche intitulée TransEnergy financée par l'ANR dans le cadre du programme Villes Durables ${ }^{1}$ (Ortar et al., 2014). Après avoir justifié le choix d'une enquête qualitative (A), nous présenterons le profil des entreprises enquêtées (B) ainsi que les terrains d'enquête (C).

\section{A. Le choix d'une enquête qualitative}

L'un des objectifs de la recherche TransEnergy était de voir comment les entreprises envisageaient la mutation énergétique dans le domaine de la mobilité et quelles étaient les stratégies développées pour y faire face. Pour recueillir le point de vue des entreprises, deux méthodes pouvaient être utilisées : une enquête quantitative basée sur la passation d'un questionnaire auprès d'un échantillon représentatif de chefs d'entreprises ou une enquête qualitative fondée sur la réalisation d'entretiens semi-directifs auprès d'un échantillon restreint de chefs d'entreprises. L'enquête quantitative a été écartée car le contexte auquel nous souhaitions faire référence - celui d'une mutation énergétique - apparaissait à l'époque ${ }^{2}$ trop incertain pour pouvoir construire un questionnaire : sans savoir au préalable ce qui faisait sens pour les chefs d'entreprises, il apparaissait difficile d'identifier les questions pertinentes à poser et l'absence d'interaction imposée par le questionnaire pouvait engendrer des risques d'incompréhension des questions. Par ailleurs, l'enquête qualitative paraissait plus à même de dévoiler la « vision du monde » des chefs d'entreprises : le but de cette recherche n'était pas d'obtenir une simple réponse à des questions mais bien de recueillir un discours des chefs d'entreprises. Pour cela, une vingtaine d'entretiens semi-directifs d'une durée variant de 45 minutes à plus d'une heure et demie ont été réalisés : il ne s'agissait pas de travailler à partir de grands échantillons représentatifs du point de vue de l'ensemble des chefs d'entreprises, mais bien de comprendre en profondeur ces points de vue à partir d'un nombre restreint d'entretiens approfondis. Ceux-ci consistaient à aborder des thématiques générales (stratégie de localisation de l'entreprise, politique de développement durable, importance du transport dans l'activité de l'entreprise) et à voir si, à un moment ou à un autre, des préoccupations particulières en matière de mobilité surgissaient en lien avec la thématique de la mutation énergétique (augmentation du prix du pétrole, réduction des émissions de gaz à effet de serre, raréfaction des ressources énergétiques fossiles). A chaque fois, nous nous sommes efforcés d'interroger le «plus haut niveau », c'est-à-dire le chef d'entreprise 
lorsqu'elle celle-ci était composée d'un seul établissement ou le responsable de l'établissement lorsque l'entreprise était organisée en plusieurs établissements. Ceux-ci nous semblaient plus à même de parler de ces enjeux que les directeurs de ressources humaines ou les responsables qualité-environnement qui risquaient d'en avoir une vision plus partielle. L'accès aux chefs d'entreprises s'est fait de manière directe en contactant les entreprises par téléphone et de manière indirecte en passant par l'intermédiaire d'élus locaux, de techniciens des collectivités locales, des CCI ou d'autres chefs d'entreprises. Contre toute attente, les chefs d'entreprises ont fait très peu de difficultés pour nous recevoir et ont manifesté un fort intérêt pour les échanges que nous avons pu avoir.

\section{B. Présentation des entreprises enquêtées}

Pour identifier les entreprises potentiellement concernées par cette mutation énergétique dans le domaine de la mobilité, un premier volet de la recherche TransEnergy avait consisté à déterminer, au sein de l'aire urbaine lyonnaise, les caractéristiques des entreprises influant sur les émissions de $\mathrm{CO}_{2}$ liées aux mobilités (secteur d'activité, taille...) ainsi que les lieux d'activités les plus émetteurs (Bouzouina et al., 2013). Il s'agissait essentiellement d'entreprises localisées dans des communes périurbaines de l'aire urbaine lyonnaise, de plus de 50 salariés, appartenant aux secteurs de l'industrie de biens intermédiaires, du commerce de gros, des services aux entreprises, du transport et de la logistique. L'objectif de l'enquête qualitative, qui constituait le deuxième volet de la recherche, était de recueillir le discours des dirigeants de ces entreprises qui pouvaient à priori être concernés par cette mutation énergétique dans le domaine de la mobilité. Pour pouvoir rendre compte de la variété des points de vue des chefs d'entreprises, l'échantillon a été constitué en diversifiant le plus possible le profil des entreprises à partir des trois variables explicatives identifiées : le secteur d'activité, la taille et la localisation de l'entreprise.

Tableau 1 : Présentation de l'échantillon d'entreprises enquêtées

\begin{tabular}{|l|c|c|c|}
\hline Communes & Nombre d'entreprises & Secteurs d'activité & Tailles \\
\hline Limonest & 4 & $\mathrm{CG} / \mathrm{IBI} / \mathrm{TL}$ & $\mathrm{PME} / \mathrm{ETI}$ \\
\hline Saint-Vulbas & 3 & $\mathrm{IBI} / \mathrm{TL} / \mathrm{SE}$ & $\mathrm{PME} / \mathrm{ETI}$ \\
\hline Saint-Quentin-Fallavier & 5 & $\mathrm{CG} / \mathrm{IBI} / \mathrm{SE}$ & $\mathrm{PME}$ \\
\hline Genas-Pusignan & 4 & $\mathrm{TL} / \mathrm{SE}$ & $\mathrm{PME}$ \\
\hline Corbas & 4 & $\mathrm{CG} / \mathrm{IBI} / \mathrm{SE}$ & $\mathrm{PME} / \mathrm{ETI}$ \\
\hline
\end{tabular}

Les entreprises enquêtées appartiennent aussi bien aux secteurs de l'industrie de biens intermédiaires (IBI), du commerce de gros (CG), des services aux entreprises (SE), que du transport et de la logistique (TL), secteurs traditionnellement surreprésentés dans les territoires périurbains. Elles comptent de 49 à 700 salariés et se rattachent donc à la catégorie des petites et moyennes entreprises (PME) comme des entreprises de taille intermédiaire (ETI). Il s'agit, soit de sièges sociaux d'entreprises disposant d'un ou de plusieurs établissements en France ou à l'étranger, soit d'établissements appartenant à des entreprises dont le siège social est situé à l'étranger (Allemagne, Etats-Unis, Royaume-Uni, Suisse). Elles sont localisées dans des communes périurbaines de la métropole lyonnaise, au sein de zones d'activités économiques ou dans le diffus.

Les entreprises enquêtées sont très dépendantes de la mobilité routière, aussi bien pour le transport de marchandises que pour les déplacements professionnels ou les déplacements domicile-travail de leurs salariés. Le transport routier de marchandises joue un rôle souvent essentiel dans l'activité de ces entreprises : c'est le cas, bien entendu, des entreprises appartenant au secteur du transport et de la logistique qui ont une couverture plutôt régionale (agglomération lyonnaise, région Rhône-Alpes, Sud-Est de la France), mais aussi des entreprises appartenant à d'autres secteurs. Celles-ci reçoivent ou expédient des produits à l'échelle régionale, nationale, voire européenne par l'intermédiaire de sous-traitants ou de leur propre flotte de camions. Par ailleurs, l'activité de certaines entreprises nécessite d'importants déplacements professionnels (commerciaux, techniciens, déplacements vers d'autres établissements) qui impliquent parfois pour l'entreprise de posséder sa propre flotte 
de véhicules. Enfin, la plupart des salariés de ces entreprises sont dépendants de la voiture pour se rendre sur leur lieu de travail situé dans des territoires périurbains mal desservis par les transports collectifs. Ils parcourent en moyenne 25 kilomètres pour se rendre sur leur lieu de travail, ce qui est largement supérieur à la moyenne des actifs de la métropole lyonnaise (8 kilomètres). Ils habitent, soit dans l'agglomération lyonnaise, soit dans le périurbain au sein de l'aire urbaine lyonnaise voire en dehors de celle-ci. Il s'agit de salariés peu qualifiés qui appartiennent aux catégories des ouvriers (opérateurs, manutentionnaires, préparateurs de commande), des professions intermédiaires (techniciens, agents de maitrise) et des employés (secrétaires, commerciaux, agents de transit) qui travaillent souvent au rythme des $2 \times 8$ ou des $3 \times 8$.

\section{Présentation des terrains enquêtés}

La plupart des terrains enquêtés sont situés dans la deuxième couronne de la métropole lyonnaise, à l'est, où se concentre une part importante de l'activité économique. Ils offrent l'image d'entrepôts, d'usines, de bâtiments tertiaires s'étendant à perte de vue dans un paysage de plaine sillonné par de larges infrastructures routières sur lesquelles circule un trafic intense, au milieu de terrains agricoles et de lotissements pavillonnaires. Afin de diversifier les localisations, six terrains ont été choisis en fonction des enjeux qu'ils représentaient en matière d'économie et de transport.

Les terrains enquêtés constituent des pôles économiques majeurs au sein de la métropole lyonnaise. Les deux premiers terrains sont situés aux franges de l'agglomération lyonnaise, à une quinzaine de kilomètres de Lyon : il s'agit de la commune de Corbas, spécialisée dans l'agro-alimentaire et la distribution, et de la commune de Limonest qui abrite une partie du parc d'affaire tertiaire Techlid, le pôle économique de l'Ouest lyonnais. Les troisième et quatrième terrains correspondent aux communes de Genas et de Pusignan situées à 25 kilomètres à l'est de Lyon, à proximité de l'aéroport Lyon-Saint Exupéry. Elles abritent un tissu d'entreprises implantées, soit dans le diffus, soit dans de petites zones d'activités économiques. Enfin, les cinquième et sixième terrains sont situés relativement loin de l'agglomération lyonnaise, à une quarantaine de kilomètres à l'est : il y la commune de Saint-Vulbas qui abrite une partie du Parc Industriel de la Plaine de l'Ain (PIPA) spécialisé dans l'industrie chimique et la logistique et la commune de Saint-Quentin-Fallavier sur laquelle est implantée une partie du Parc international de Chesnes spécialisé dans la logistique et le commerce de gros. Ces pôles économiques sont intégrés à un réseau routier et autoroutier très dense (boulevard urbain, périphérique, rocade, autoroutes) qui leur permet de bénéficier d'une excellente accessibilité routière. L'offre en transports alternatifs (transports collectifs, covoiturage, frêt ferroviaire ou fluvial) est, par contre, soit inexistante, soit peu performante (horaires, fréquences, durées inadaptées), imposant, de fait, le recours aux modes routiers.

\section{Mobilité et mutation énergétique : quels enjeux pour les entreprises implantées dans les territoires périurbains ?}

Les chefs d'entreprises qui ont fait le choix de s'implanter dans ces territoires périurbains en raison notamment de la forte accessibilité routière qu'ils offraient sont-ils préoccupés par la mutation énergétique qui s'annonce dans le domaine de la mobilité ? Quels types de stratégies ont-ils développé pour y faire face ? Quel que soit le secteur d'activité, la taille ou la localisation de l'entreprise, on constate dans les discours des chefs d'entreprises une réelle préoccupation quant à cette question. Mais cet intérêt semble essentiellement lié à la première conséquence perceptible de cette mutation énergétique, la hausse des prix du pétrole et donc du coût de la mobilité routière, qui apparaît porteuse pour ces entreprises d'enjeux économiques (A) et sociaux importants (B). Par contre, la réduction des émissions de $\mathrm{CO}_{2}$ générées par la mobilité des biens et des personnes, affichée par les pouvoirs publics comme un enjeu environnemental prioritaire, apparait secondaire voire inexistante aux yeux des chefs d'entreprises n'appartenant pas au secteur du transport et de la logistique (C). 


\section{A. L'augmentation du coût de la mobilité : un enjeu économique fort pour les entreprises}

La mutation énergétique dans le domaine de la mobilité apparaît, d'abord, porteuse d'enjeux économiques forts pour les chefs d'entreprises enquêtés. Les hausses du prix du pétrole, et donc du prix du carburant, se sont traduites par une augmentation significative des coûts de la mobilité pour ces entreprises dont l'activité est fortement dépendante de la mobilité routière, que ce soit à travers le transport de marchandises ou les déplacements professionnels de leurs salariés.

C'est le cas, bien sûr, des entreprises du transport et de la logistique dont c'est le cœur d'activité. Pour elles, les hausses des prix du carburant ont eu un impact énorme, les charges liées à la consommation de gasoil représentant une part importante de leur coût de revient. Les chefs d'entreprises considèrent que s'ils n'avaient pas été autorisés à répercuter les évolutions du prix du gasoil sur le prix de leurs prestations, ils auraient dû déposer le bilan comme l'ont d'ailleurs fait beaucoup d'entreprises n'ayant pas effectué ces refacturations. Mais c'est le cas également des entreprises appartenant à d'autres secteurs d'activité pour qui la hausse des prix du carburant s'est également traduite par une augmentation significative de leurs coûts de mobilité, soit parce qu'elles ont vu le prix des prestations de leurs sous-traitants augmenter, soit parce qu'elles ont dû elles-mêmes faire face à une augmentation des charges liées à la possession d'une flotte de véhicules (camions pour réaliser des livraisons locales, voitures pour les déplacements professionnels des commerciaux...).

Enquêté $\mathrm{P}$, entreprise du secteur de l'industrie de biens intermédiaires, Limonest : «L'augmentation du prix de l'essence et du pétrole, il y a un enjeu important dans la mesure où l'on $\mathrm{a}$, comme je vous l'ai dit, une grande partie de la population employée qui est itinérante. Avec les hausses actuelles des prix à la pompe, il y a une incidence importante. On essaie de répercuter, pour partie, des surcharges carburant à nos clients. On a un enjeu important à ce niveaulà, oui. [...]. Mais c'est sûr que forcément on devra se remettre en question sur nos façons de nous déplacer.»

Bien que les fluctuations du prix du pétrole dessinent un univers relativement incertain, les chefs d'entreprises rencontrés considèrent que la hausse des prix du carburant est un phénomène durable qui s'inscrit dans un renchérissement général des prix de l'énergie (gaz, électricité...) et qui se traduira inévitablement par une augmentation des coûts de la mobilité dans les années à venir. A ce titre, ils ont déjà mis en œuvre des stratégies destinées à faire évoluer les pratiques de mobilité au sein de leur entreprise. Celles-ci consistent essentiellement à réduire le coût de la mobilité routière, soit en optimisant les déplacements routiers, soit en réduisant les consommations de carburant. L'optimisation des déplacements routiers consiste, par exemple, à éviter les trajets à vide ou à augmenter le taux de remplissage des camions ou à optimiser les tournées de livraison pour le transport de marchandises, à favoriser le covoiturage pour les déplacements professionnels. La diminution des consommations de carburant passe, quant à elle, par l'achat de véhicules aux normes Euro 5, le suivi de la consommation des conducteurs, le financement de stages de formation à l'éco-conduite, le choix de nouveaux types de carburants, pour les déplacements professionnels comme pour le transport de marchandises.

Enquêté G, entreprise du secteur de l'industrie de biens intermédiaires, Saint-Quentin-Fallavier : «L'augmentation du prix de l'énergie c'est un enjeu, bien sûr! Au niveau de l'électricité, c'est énorme. Du gasoil aussi, forcément, vu que l'on transporte nos produits. [...]. Actuellement, l'enjeu c'est la chasse au kilomètre parasitaire ! Optimisation des coûts, réduction des consommations de carburant ; les trajets au plus court, les plus sûrs et les plus courts. C'est surtout ça ; c'est vraiment réduire la consommation de carburant, de par la formation des conducteurs pour qu'ils adoptent une conduite plus économique et par les camions qu'on utilise aussi qui doivent être performants. [...]. On a des objectifs qui sont fixés pour les coûts de transport, à nous de les tenir et d'être en dessous, si possible. » 


\section{B. L'augmentation du coût de la mobilité : un enjeu social fort pour les entreprises}

La mutation énergétique dans le domaine de la mobilité apparaît, également, porteuse d'enjeux sociaux importants pour les chefs d'entreprises enquêtés. Les entreprises localisées dans ces territoires périurbains étant souvent des sites de production ou des sites logistiques, elles emploient en grande majorité des salariés à faible qualification qui disposent de revenus peu élevés. Il s'agit principalement d'ouvriers qui travaillent aux trois-huit dans les secteurs de l'industrie (ouvriers qualifiés, techniciens de laboratoires, ouvriers de production...) ou du transport et de la logistique (chauffeurs, agents de manutention, magasiniers, préparateurs de commande...) et d'employés qui effectuent un travail administratif au sein de l'entreprise (secrétariat, comptabilité, informatique...). Elles emploient également beaucoup d'intérimaires et de stagiaires dont le nombre varie selon l'évolution des besoins des entreprises. L'augmentation du coût de la mobilité automobile liée aux hausses des prix $\mathrm{du}$ carburant pèse sur le budget de ces salariés qui disposent de revenus modestes, habitent souvent loin de leur lieu de travail et parcourent, en moyenne, 50 kilomètres par jour. Des chefs d'entreprises ont été interpellés par les syndicats sur cette question de l'augmentation du coût des déplacements domicile-travail pour les salariés liée à la hausse des prix du carburant et certains doivent même faire face à des problèmes de recrutement ou de turn-over.

Enquêté R, entreprise du secteur du commerce de gros, Limonest : « La plupart des salariés voient leur pouvoir d'achat se réduire en fonction, fatalement, du coût du prix à la pompe. Oui, via les instances représentatives du personnel, oui ça remonte, oui c'est clair. [...]A un moment donné, c'était monté tellement haut, les gens étaient tellement angoissés, qu'on se disait même est-ce qu'un jour il ne va pas falloir payer les gens pour qu'ils viennent travailler?»

Pour faire face à l'augmentation du coût de la mobilité domicile-travail des salariés, les entreprises ont développé plusieurs stratégies : certains essayent de favoriser un recrutement de proximité, d'autres de proposer des modes de transport alternatifs à travers la mise en place de systèmes plus ou moins formels de covoiturage, d'adhérer à des plans de déplacements interentreprises (PDIE). Mais la plupart du temps, la stratégie la plus couramment adoptée par les chefs d'entreprises enquêtés consiste à subventionner la mobilité automobile, à travers la prise en charge par l'entreprise d'une partie des frais de déplacements domicile-travail effectués en voiture, ce qui est loin d'aller dans le sens d'une réduction des émissions de $\mathrm{CO}_{2}$ générées pas les mobilités.

Enquêté A, entreprise du secteur de l'industrie de biens intermédiaires, Saint-Vulbas : «Cette année, ce que je vais proposer pour la première fois dans le cadre des négociations salariales, c'est un coupon de rémunération lié aux transports. C'est une première. Je pense que les gens y sont favorables, forcément, c'est un peu d'argent donc... [...] On aura trois zones. Et les gens, s'ils sont bien sûr dans l'une des trois zones et en fonction de là où ils sont, ils auront un complément de rémunération. [...].C'est pour prendre en compte le fait, effectivement de... on va dire des coûts croissant, liés aux déplacements. C'est vrai que l'on avait estimé que... je me rappelle... quand on avait fait notre étude, ça peut représenter quasiment un mois de la rémunération de quelqu'un. Le budget transport, c'est entre 10-15\%. Pour un petit salaire, ça peut s'élever entre 10-15\% ; c'està-dire, un mois de rémunération. C'est quand même des choses importantes. »

\section{La réduction des émissions de $\mathrm{CO}_{2}$ générées par les mobilités : un faible enjeu environnemental pour les entreprises}

17 La mutation énergétique dans le domaine de la mobilité n'apparaît pas, par contre, porteuse d'un enjeu environnemental important dans les discours des chefs d'entreprises enquêtés. Cette attitude ne signifie pas pour autant que les chefs d'entreprises se désintéressent de la question environnementale. Au contraire même, ces entreprises mènent des politiques plutôt actives en matière de développement durable : avec l'attention grandissante portée à la responsabilité sociale et environnementale des entreprises (Bost, 2011) ces politiques sont devenues indispensables à l'image de l'entreprise et elles permettent, en outre, de réaliser des économies au sein de l'entreprise.

18 Mais les politiques de développement durable menées par les entreprises concernent, soit l'activité même de l'entreprise, soit des domaines annexes (déchets, eau, air) dont la 
mobilité ne fait pas partie. Lorsque ces entreprises sont invitées à parler de leur politique environnementale elles évoquent spontanément les mesures liées au tri des déchets, au traitement des eaux, au recyclage du papier, aux politiques d'efficacité énergétique (éteindre les lumières, les ordinateurs, limiter l'utilisation de la climatisation...). Mais elles ne mentionnent jamais les stratégies mises en place pour faire évoluer les pratiques de mobilité au sein de l'entreprise, comme si celles-ci étaient déconnectées d'enjeux environnementaux. La mobilité apparaît comme l'angle mort des politiques de développement durable des entreprises : l'impact négatif sur l'environnement du transport de marchandises, des déplacements professionnels, des déplacements domicile-travail engendrés par l'entreprise apparaissent encore comme des externalités négatives liées à l'activité de l'entreprise, sur lequel sa responsabilité n'est pas engagée et dont globalement les chefs d'entreprises ont peu conscience (Enoch, 2012). Seule exception à la règle, les entreprises de transport et de logistique dont la mobilité constitue le cœur d'activité. Pour ces entreprises, les stratégies développées pour optimiser les déplacements routiers ou réduire les consommations de carburant sont présentées dans les discours des chefs d'entreprises comme porteuses d'un enjeu économique mais aussi environnemental. Dans ce contexte de mutation énergétique, c'est un moyen pour les chefs d'entreprises d'améliorer leur image en montrant qu'ils cherchent à réduire leurs émissions de $\mathrm{CO}_{2}$.

\section{Mutation énergétique, mobilité et périurbanisation économique}

19 Dans quelle mesure l'émergence de ces enjeux économiques et sociaux liés à la mutation énergétique dans le domaine de la mobilité modifie-t-elle le rapport des entreprises à leur territoire d'implantation ? La localisation périurbaine pourrait-elle, à l'avenir, poser des problèmes aux entreprises? Si l'implantation dans les territoires périurbains ne semble pas poser de problème par rapport aux enjeux économiques soulevés par la mutation énergétique qui s'annonce dans le domaine de la mobilité, elle en pose, par contre, par rapport aux enjeux sociaux. Les difficultés rencontrées par les salariés face à l'augmentation du coût de la mobilité semblent faire évoluer le discours des chefs d'entreprises sur les questions de l'éloignement croissant des agglomérations (A) et de l'offre en transports alternatifs dans les territoires périurbains (B).

\section{A. La question de l'éloignement croissant des agglomérations}

L'éloignement des agglomérations constitue pour les entreprises implantées dans des territoires périurbains un des avantages essentiels de cette localisation. Il procure à ces entreprises fortement consommatrices d'espace des disponibilités foncières très importantes à un prix peu élevé, leur permettant à la fois de répondre à leurs besoins en surface actuels mais aussi futurs en leur offrant des possibilités d'extension. Il leur permet également de bénéficier d'une forte accessibilité routière sans pour autant souffrir des problèmes de congestion. Ces éléments poussent les entreprises à s'implanter dans des territoires périurbains de plus en plus lointains (CERTU, 2011), renforçant ainsi l'étalement économique (Demazière, 2012).

Mais, avec la montée des enjeux sociaux liés à l'augmentation du coût de la mobilité automobile, cet éloignement croissant pourrait devenir un inconvénient pour certaines entreprises mais aussi pour certains territoires, en raison de la faiblesse de leur bassin d'emploi. Certains chefs d'entreprises interrogés, implantés dans des territoires périurbains situés à une quarantaine de kilomètres de l'agglomération lyonnaise, semblent ainsi rencontrer des problèmes de turn-over et de recrutement en raison de l'augmentation du coût de la mobilité automobile, les salariés faiblement qualifiés ne postulant plus sur des postes trop éloignés en raison de coûts de mobilité trop élevés.

Enquêté D, entreprise du secteur du transport et de la logistique, Saint-Vulbas : «L'inconvénient de notre localisation c'est qu'on a un bassin d'emploi plus faible avec un taux de chômage très bas. La proximité lieu de travail-lieu de résidence, c'est une vraie préoccupation avec l'augmentation du prix du pétrole, c'est dur pour les salariés, le budget voiture est très important. Certains de nos salariés qui résidaient à Lyon ont même dû démissionner car c'était trop coûteux. On a des difficultés de recrutement également, les gens ne veulent pas venir de Lyon, c'est trop loin... » 
D'ailleurs, parmi les entreprises enquêtées implantées récemment, certaines semblent avoir mis en œuvre des stratégies de localisation plus sélectives : certains territoires périurbains semblent désormais évités par les entreprises car elles savent que l'éloignement risque de poser des problèmes d'accessibilité des salariés à leur lieu de travail, en raison de l'augmentation du coût de la mobilité.

Enquêté I, entreprise du secteur des services aux entreprises, Pusignan : « Non, non, il n’y a pas d'autres zones de l'agglomération qui pourraient être plus attractives. Clairement non, parce que d'autres zones qui par le passé ont essayé de se développer, type Saint-Quentin-Fallavier, type Saint-Vulbas, etc., et bien on se rend compte qu'elles ont des limites. Même si c'est beaucoup moins cher qu'ici en termes de foncier, le prix au mètre carré est moins cher, mais il y a de gros problèmes de recrutement de personnel, il y a de gros problèmes d'accessibilité, etc. Ce qui fait que c'est peut-être d'un premier abord plus intéressant, mais très vite... C'est sûr que le foncier au fin fond de la plaine de l'Ain est moins cher. Mais bon, si vous n'arrivez pas à recruter de personnel ou si vous devez le rémunérer deux fois plus pour le faire venir, ça pose des problèmes aussi. »

Si la mutation énergétique qui s'annonce dans le domaine de la mobilité ne semble pas remettre en cause l'attrait des entreprises pour les territoires périurbains, elle pourrait par contre peutêtre constituer un frein à l'étalement économique, dans la mesure où l'implantation dans des territoires périurbains de plus en plus éloignés, disposant d'un faible bassin d'emploi, risque à terme de poser des problèmes de recrutement et de turn-over pour les entreprises.

\section{B. La question de l'offre en transports alternatifs des territoires périurbains}

La faiblesse de l'offre en transports alternatifs n'a, jusqu'à présent, jamais constitué un inconvénient de localisation pour les entreprises implantées dans les territoires périurbains. La dépendance à la mobilité routière des territoires périurbains était même recherchée dans la mesure où elle présentait le double avantage de répondre aux besoins de mobilité de l'entreprise (transport de marchandises, déplacements professionnels), mais aussi de ses salariés, la diffusion de l'automobile permettant potentiellement à tout salarié d'accéder à son lieu de travail, quel que soit son lieu de résidence.

Les choses seraient-elles en train de changer sous l'effet de la montée des enjeux sociaux liés à l'augmentation du coût de la mobilité automobile ? Dans le cadre des entretiens menés, nous avons pu constater que la faiblesse de l'offre en transports alternatifs des territoires périurbains constituait un sujet de préoccupation pour la plupart des chefs d'entreprises enquêtés. C'est, d'ailleurs, le premier inconvénient de localisation cité par les chefs d'entreprises qui ont fait le choix de s'implanter dans ces territoires périurbains. Enquêté $\mathrm{H}$, entreprise du secteur des services aux entreprises, Pusignan : «L'inconvénient c'est
l'accès en transports urbains, puisqu'il y a très peu de possibilités ou, autant dire, pas de possibilité
pour les salariés de venir ici avec un transport urbain. [...]. Au niveau des transports en commun,
on est pénalisés. Et c'est très pénalisant pour les salariés, surtout avec l'augmentation du prix
de l'énergie. Ils aimeraient que les transports en commun soient présents pour pouvoir éviter de
prendre la voiture. [...]. On a des demandes de réajustement salarial pour pallier l'augmentation
de l'énergie et des problèmes de recrutement pour les postes de base, oui. C'est-à-dire, les ouvriers
ou les préparateurs de commande. C'est une problématique. »

La mutation énergétique qui s'annonce dans le domaine de la mobilité apparaît porteuse de deux évolutions importantes du positionnement des entreprises sur cette question de l'offre en transports alternatifs des territoires périurbains. D'une part, les chefs d'entreprises semblent porter un intérêt pour le développement d'une offre en transports alternatifs dans les territoires périurbains alors qu'ils s'en souciaient peu jusqu'à présent (Mérenne-Schoumaker, 2008a et 2008b). D'autre part, ils semblent porter une attention nouvelle à la question de la mobilité de leurs salariés, domaine dont ils s'étaient progressivement désintéressés avec la diffusion de l'automobile (Moati \& Van de Walle, 2002), après être intervenus intensément dans les années soixante à travers l'organisation de services de ramassage (Gérardin, 1981).

Le rapport des entreprises aux territoires périurbains semble évoluer sous l'effet de l'émergence des enjeux sociaux liés à la mutation énergétique qui s'annonce dans le domaine de la mobilité. Avec l'augmentation prévisible du coût de la mobilité pour les salariés, il 
semblerait, d'après les résultats de cette enquête, que les entreprises désireuses de s'implanter dans des territoires périurbains développent à l'avenir un intérêt moindre pour les territoires périurbains les plus éloignés disposant d'un faible bassin de main d'œuvre et, parallèlement, un intérêt grandissant pour les territoires périurbains proposant une offre en transports alternatifs. Autant d'éléments qui pourraient constituer une réponse, bien que partielle, aux enjeux environnementaux associés à cette mutation énergétique.

\section{Conclusion}

Pour les entreprises implantées dans les territoires périurbains de la métropole lyonnaise et fortement dépendantes de la mobilité routière, la mutation énergétique qui s'annonce dans le domaine de la mobilité apparaît porteuse d'enjeux économiques et sociaux, mais rarement environnementaux. Les chefs d'entreprises apparaissent plus préoccupés par l'augmentation du coût de la mobilité liée à la hausse du prix de l'énergie, qui pèse sur l'activité de l'entreprise et sur le budget des salariés, que par la réduction des émissions de $\mathrm{CO}_{2}$ qu'ils génèrent à travers le transport de marchandises, les déplacements professionnels et les déplacements domiciletravail de leurs salariés. Pour faire face à ces enjeux, les chefs d'entreprises ont développé des stratégies contradictoires : ils cherchent à réduire le coût des mobilités générées par leur activité, mais ils subventionnent la mobilité automobile de leurs salariés. Si l'émergence de ces enjeux, en particulier sociaux, ne remet pas en cause l'attrait de ces chefs d'entreprises pour les territoires périurbains, elle contribue à faire évoluer leur rapport à ces territoires : les chefs d'entreprises semblent moins enclins à se localiser dans des territoires périurbains très éloignés en raison de la faiblesse de leur bassin d'emploi et plus demandeurs du développement d'une offre en transports alternatifs dans ces territoires. Ces résultats n'apportent naturellement qu'une vision partielle des stratégies développées par les entreprises pour faire face à la mutation énergétique qui s'annonce dans le domaine de la mobilité : il serait intéressant de le compléter en s'intéressant à d'autres profils d'entreprises et à d'autres types de localisations.

\section{Bibliographie}

Aguilera A., 2008, « Business travel and mobile workers », Transportation Research Part A, Vol. 42, p.1109-1116.

Aguiléra A., De Coninck F., Hauchard P., 2007, « Le rôle des déplacements professionnels dans les entreprises industrielles multi-établissements. Le cas d'un fournisseur de l'automobile », Recherche Transports Sécurité, Vol. 24, n96, p. 195-209.

Association Négawatt, 2012, Manifeste Négawatt. Réussir la transition énergétique, Arles, Actes Sud, $368 \mathrm{p}$.

Blanchet A., Gotman A., 1992, L'enquête et ses méthodes : l'entretien, Paris, Nathan, 125 p.

Bonnet J., Broggio C., 2010, Entreprises et territoires, Paris, Ellipses, 240 p.

Bost F., Daviet S. (dir.), 2011, Entreprises et environnement : quels enjeux pour le développement durable ?, Nanterre, Presses universitaires de Paris Ouest, 360 p.

Bourg D., Grandjean A., Libaert T. (dir.), 2006, Environnement et entreprises : en finir avec les discours, Paris, Village mondial, $250 \mathrm{p}$.

Bouzouina L, Quetelard B., Toilier F., 2013, «Emissions de $\mathrm{CO}_{2}$ liées à la mobilité domicile-travail : une double lecture par le lieu de résidence et par le lieu de travail des actifs à Lyon et à Lille », Développement durable et Territoires (en ligne), Vol.4, n³, mis en ligne le 30 octobre 2013, consulté le 03 février 2014, URL :http://developpementdurable.revues.org/10018

Chamboredon H., Pavis F., Surdez M., Willemez L., 1994, « S'imposer aux imposants. A propos de quelques obstacles rencontrés par des sociologues débutants dans la pratique et l'usage de l'entretien », Genèses, n¹6, p. 114-132.

CERTU, 2011, Le desserrement des activités économiques. Localisation de l'évolution de l'emploi salarié privé entre 1997 et 2008, Lyon, CERTU, 8 p.

Demazière C., 2007, «Les entreprises peuvent-elles promouvoir le développement durable ? », Les cahiers français, $\mathrm{n}^{\circ} 37$, p. 83-89. 
Demazière C. (dir.), 2012, Viabilité de l'économie productive des régions urbaines : investigation à partir de la planification stratégique. Une comparaison entre la France et l'Angleterre, rapport final pour le PUCA, Tours (Citères), 415 p.

Enoch M., 2012, Sustainable Transport, Mobility Management and Travel Plans, Farnham, Ashgate Publishing, $225 \mathrm{p}$.

Gérardin B., 1981, Le transport employeur, Paris, Economica, 200 p.

Hayter R., Patchell J. (dir.), 2011, Economic Geography: an Institutional Approach, London, Oxford University Press, 464 p.

IEA, 2013, Key World Energy Statistics, Paris, IEA, 82 p.

IEA, 2009, Transport, Energy and $\mathrm{CO}_{2}$, Paris, IEA, 418 p.

Knowles R. D., Shaw J., Docherty I. (dir.), 2008, Transport geographies : mobilities, flows and spaces, Malden, Blackwell Publishing, 293 p.

Le Breton E., 2008, Domicile-travail : les salariés à bout de souffle, Paris, Les carnets de l'info, 216 p.

Lejoux P., Ortar N., 2014, « La transition énergétique : vrais enjeux, faux départs ? » in Lejoux P., Ortar N., La transition énergétique : vrais enjeux, faux départs?, SHS Web of Conferences (en ligne), Vol. 9, $\mathrm{n}^{\circ} 01001$, mis en ligne le 4 juillet 2014, consulté le 15 juillet 2014, URL : http://dx.doi.org/10.1051/ shsconf/20140901001

Lejoux P., 2014, Entreprises et mobilités durables en territoires périurbains. Le cas du bassin annecien, rapport final pour le CEREMA, Lyon (LET), 34 p.

Mérenne-Schoumaker B., 2008a, La localisation des industries. Enjeux et dynamiques, Rennes, PUR, $255 \mathrm{p}$.

Mérenne-Schoumaker B., 2008b, Géographie des services et des commerces, Rennes, PUR, 255 p.

Moati P., Van De Walle I., 2002, Mobilités et territoires urbains. Les stratégies économiques et territoriales des entreprises à l'égard de la mobilité vers le travail, rapport final pour le PUCA, Paris (CREDOC), $135 \mathrm{p}$.

Orfeuil J.-P., 2008, Une approche lä̈que de la mobilité, Paris, Descartes \& Cie, 173 p.

Ortar N. (dir.), 2014, Les stratégies d'adaptation des ménages et des entreprises face à la transition énergétique : une comparaison entre les métropoles de Lille et de Lyon, rapport final pour l'ANR, Lyon (LET), $130 \mathrm{p}$.

Quételard B., 2010, «Se rendre au travail ou faire ses courses motive toujours un déplacement quotidien sur deux. Le recours à la voiture se stabilise », La Revue du CGDD, p. 25-48.

Rifkin J., 2012, La troisième révolution industrielle. Comment le pouvoir latéral va transformer l'énergie, l'économie et le monde, Paris, Les liens qui libèrent, 411 p.

Rodrigue J.-P., Comtois C., Slack B. (dir.), 2013, The Geography of Transport Systems, London, Routledge, $352 \mathrm{p}$.

Ison S., Rye T. (dir.), 2008, The Implementation and Effectiveness of Transport Demand Management Measures, Farnham, Ashgate Publishing, 262 p.

Van Malderen L., Jourquin B., Thomas I., Vanoutrive T., Verhetsel A., Witlox F., 2012, « The mobility policies of companies: What are the good practices? The Belgian case », Transport Policy, Vol. 21, p. $10-19$.

\section{Notes}

1 Cette recherche a été menée par le LET (CNRS, ENTPE, Université Lyon 2), en partenariat avec le CLERSE (CNRS, Université Lille 1), le LTE (IFSTTAR), le CETE Nord-Picardie, le Grand Lyon et Lille Métropole.

2 Au moment où les entretiens ont été menés (printemps 2011 et 2012), la question de la transition énergétique n'était pas encore d'actualité. Celle-ci est véritablement apparue dans le débat public à l'occasion de la Conférence environnementale sur le développement durable organisée par l'Etat en septembre 2012 


\section{Pour citer cet article}

Référence électronique

Patricia Lejoux, «Les entreprises face à la mutation énergétique : l'enjeu de la mobilité dans les territoires périurbains de la métropole lyonnaise », Revue Géographique de l'Est [En ligne], vol. 54 / 1-2 | 2014, mis en ligne le 15 octobre 2014, consulté le 31 octobre 2014. URL : http:// rge.revues.org/5135

\section{À propos de l'auteur}

Patricia Lejoux

Laboratoire d'Economie des Transports, Ecole Nationale des Travaux Publics de l'Etat - 3 rue Maurice Audin, 69518 Vaulx-en-Velin cedex - patricia.lejoux@entpe.fr

\section{Droits d'auteur}

Tous droits réservés

\section{Résumés}

Dans une économie mondialisée, la mobilité est devenue indispensable au fonctionnement des entreprises : le transport de marchandises, les déplacements professionnels, les déplacements domicile-travail des salariés permettent à l'entreprise-réseau d'être connectée à tous les territoires. Mais dans un contexte marqué par la raréfaction prévisible des énergies fossiles et la nécessité de réduire les émissions de gaz à effet de serre, comment les entreprises envisagent-elles la mutation énergétique qui s'annonce dans le domaine de la mobilité ? Une enquête menée auprès d'entreprises implantées dans les zones d'activités périurbaines de la métropole lyonnaise montre que si ces entreprises apparaissent peu concernées par les enjeux environnementaux associés à cette mutation énergétique, elles semblent par contre beaucoup plus préoccupées par ses enjeux économiques et sociaux, et notamment par l'augmentation des coûts de transport pour l'entreprise comme pour ses salariés. Face à cette mutation énergétique, ces entreprises n'envisagent pas pour autant de modifier leur stratégie de localisation, même si certaines zones d'activité périurbaines trop éloignées apparaissent désormais moins attractives.

\section{Companies and energy transition: the mobilities at stake in suburban areas of the french city of Lyon}

In a globalized economy, the mobility became essential for companies: the transportation of goods, business travels, commuting allow the company to be connected to all territories. But in a context marked by the predictable rarefaction of the fossil fuels and the necessity of reducing greenhouse gas emissions, how companies envisage the energy transition announced in mobilities? A survey led with companies implanted in business parks, in the outer suburbs of the metropolitan area of Lyon, shows that if these companies seem little concerned by environmental issues associated to this energy transition, they seem on the other hand much more worried by its economic and social issues, in particular by the increase of the costs of transport for the company as for their employees. Faced to this energy transition, these companies do not intend to modify their strategy of location, even if some outer seem less attractive.

\section{Die Unternehmen und der Energiewende - das Problem der Mobilität in Lyon Stadtnahen Räume.}

In einer globalen Wirtschaft ist die Mobilität für den Betrieb der Unternehmen unabdingbar geworden. Durch Warentransporte, Geschäftsreisen, Fahrten der Beschäftigten vom Wohnort 
zum Arbeitsplatz können sich die Netzwerkunternehmen mit allen Gebieten verbinden. Vor dem Hintergrund der vorhersehbaren Verknappung der fossilen Energieträger und der Notwendigkeit der Reduzierung der Treibhausgase stellt sich die Frage, wie die Unternehmen auf den Energiewandel reagieren, der sich im Bereich der Mobilität abzeichnet. Eine bei in stadtnahen Industriegebieten der Metropole Lyon angesiedelten Unternehmen durchgeführte Umfrage hat ergeben, dass, auch wenn diese Unternehmen nur kaum von den mit dem Energiewandel einhergehenden ökologischen Problemen betroffen sind, ihnen trotzdem die ökonomischen und sozialen Herausforderungen, insbesondere durch die steigenden Transportkosten sowohl für das Unternehmen als auch für die Beschäftigten, zunehmend Sorgen zu bereiten scheinen. Angesichts des Energiewandels beabsichtigen diese Unternehmen dennoch keine Änderung ihrer Standortstrategie - dies auch dann nicht, wenn einige zu weit entfernt gelegene Industriegebiete in städtischen Randbereichen in Zukunft an Attraktivität einbüßen dürften.

\section{Entrées d'index}

Mots-clés : énergie, entreprise, localisation, mobilité, territoire

Keywords : company, energy, localization, mobility, territory

Schlagworten : Energie, Lokalisierung, Mobilität, Territorium, Unternehmen 